CIC. Cuadernos de Información y Comunicación

ISSN: $1135-7991$

\title{
Horizontes del mundo digital: de la simulación y la banalización de la experiencia, a un uso social, ecológico e innovador de la Sociedad Red
}

\author{
David Caldevilla Domínguez; ; Almudena Barrientos Báez²; Eduardo Parra López ${ }^{3}$
}

Evaluado: 08/04/2020 / Aceptado: 17/04/2020

Resumen. El presente artículo es una reflexión, al hilo de las principales tendencias de desarrollo en las aportaciones sobre la web 2.0 y la sociedad digital, que se manifiestan en dos direcciones fundamentales: la tendencia hacia la innovación económica y creativa, el desarrollo de valores y el equilibrio medioambiental, o la pulsión hacia la comercialización, manipulación y el mundo de los simulacros en sectores vitales de la actividad humana. Al hilo de las reflexiones, se plantea que desde sus orígenes la sociedad red ha tenido ante sí un panorama bipolar, en el que los acuciantes dramas políticos, económicos, ecológicos y humanos nos obligan a elegir una opción de desarrollo.

Palabras Clave: sociedad red; crisis social; simulación; innovación; equilibrio socio-ecológico; web 2.0.

\section{[en] Horizons of the digital world: from the simulation and trivialization of the experience, to a social, ecological and innovative use of the Red Society}

\begin{abstract}
This article is a reflection, in line with the main development trends in the scholar contributions on web 2.0 and the digital society, about the two fundamental directions of future development: the trend towards economic and creative innovation, and the development of values in environmental equilibrium, or the drive towards commercialization, manipulation and the fake universe in vital sectors of human activity. In line with these reflections, it states that since its origins the Network Society has met a bipolar panorama, in which pressing political, economic, ecological and human crises force us to choose a unique development option.
\end{abstract}

Keywords: Network Society; social crisis; simulation; innovation; socio-ecological balance; web 2.0.

[fr] Horizons du monde numérique: de la simulation et la trivialization de l'expérience, ver une utilisation sociale, ecologique et innovatrice de la société-reseau

Résumé: Cet article est une réflexion, en ligne avec les principales tendances des contributions critiques sur le web 2.0 et la société des médias, qui se manifestent dans deux directions fondamentales: la tendance à l'innovation économique et créative et le développement des valeurs dans un équilibre environnemental,

\footnotetext{
David Caldevilla Domínguez, Universidad Complutense de Madrid.

E-mail: dcaldevilla@ccinf.ucm.es

2 Almudena Barrientos Báez, Escuela Universitaria de Turismo Iriarte (ULL).

E-mail: almudenabarrientos@iriarteuniversidad.es

3 Eduardo Parra López, Universidad de La Laguna.

E-mail: eparra@ull.es
} 
ou bien la tendance à la commercialisation, la manipulation et le monde des simulacres dans des secteurs vitaux de l'activité humaine. Conformément aux réflexions, il est indiqué que depuis ses origines la société des reseaux a eu devant elle un panorama bipolaire, dans lequel des crises politiques, économiques, écologiques et humaines pressants nous obligent à choisir une option seule de développement.

Mots clés: société en réseau; crise sociale; simulation; innovation; équilibre socio-écologique; web 2.0.

Sumario: 1. Del nacimiento de la digitalización al planteamiento del dilema: para qué usar la Sociedad Red. 2. Sectores de un dilema: periodismo, redes sociales, innovación empresarial. 3. Nuevas tendencias de trabajo: las humanidades digitales, las ecologías sociales, los estudios medioambientales en la web 2.0. 4. Conclusiones.

Cómo citar: Caldevilla Domínguez, D.; Barrientos Báez, A.; Parra López, E. (2020). Horizontes del mundo digital: de la simulación y la banalización de la experiencia, a un uso social, ecológico e innovador de la Sociedad Red, en CIC. Cuadernos de Información y Comunicación 25, 269-277.

\section{Del nacimiento de la digitalización al planteamiento del dilema: para qué usar la Sociedad Red}

Los expertos analistas, desde planos teóricos o bien desde investigaciones socio-políticas o económicas prácticas, han marcado siempre, desde el nacimiento de Internet, hace ya más de 25 años, las dos grandes tendencias en el uso, el aprovechamiento, la gestión y la innovación de este poderoso nuevo medio en el panorama de la comunicación humana desde sus orígenes.

El dilema viene planteado desde que los primeros autores en analizar la Sociedad Red (Castells, 2005) ya disciernen que este nuevo medio, cuyas potencialidades de innovación y desarrollo social son infinitas, puede seguir dos vías bien distintas: hacia la creación y el crecimiento, o hacia la explotación comercial y el beneficio inmediato. Ambos aspectos aparecen claramente diferenciados, en tanto el denominado capitalismo cognitivo (Lessig, 2009) impone, como una prolongación de los desarrollos de las industrias culturales y de los procesos del sistema económico capitalista, sus huellas en las primeras formas de generación de procesos y productos en la red.

Al mismo tiempo que toda una estructura de inmediata obtención de beneficios, rentabilización y comercialización, va generándose en la red, a comienzos de su implantación, Internet conserva su potencial como fenómeno comunicativo surgido de la colaboración y fusión de iniciativas en continentes y en sectores de conocimiento humano dispares, de la universidad al comercio, del ejército a las telecomunicaciones. Los dos brazos de la red, el creativo y el comercial, están presentes desde su nacimiento.

Así, y en su progresivo pero acelerado avance, Internet se nos presenta como un mundo bipolar en el que asombrosos fenómenos colaborativos y creativos aparecen para generar nuevas opciones de trabajo -como en el terreno del trabajo con mayores (Caldevilla y García, 2016:162) o en el de la diversidad en educación, por citar el ejemplo de Sánchez y Martín (2016:42)- nuevas libertades de creación, nuevos entornos de innovación (Por ejemplo para PYMES, según Sánchez y De la Garza, 2018) y una fuente infinita de información que es útil para todos los sectores de 
actividad humana, vienen a ser ensombrecidos por el desmesurado crecimiento comercial de grandes empresas -en las que la imagen corporativa en Internet está desde tiempo consolidada como factor estratégico clave (Gómez, Tapia y Díaz, 2012; corroborado por los datos de Lopezosa, Codina, López y Corbella, 2020) - y grupos que acaparan la explotación de los recursos, centralizan los flujos de la nueva comunicación digital, seleccionan y condicionan las formas de innovación y creación, y finalmente, aprisionan las opciones de desarrollo en la dirección exclusiva de la obtención del beneficio para las empresas digitales en lo alto de la pirámide económica, que evaden las obligaciones fiscales, comercian con los datos de sus usuarios y restringen el acceso al conocimiento, imponiendo draconianas condiciones de explotación del mismo.

El surgimiento de la web 2.0, y la llegada de las redes sociales, no hacen sino aumentar la visibilidad de la brecha existente entre estas dos vertientes de desarrollo, que son en realidad una espada de Damocles sobre el desarrollo digital: o hacer de la red un inmenso supermercado en el que se manipulen las emociones y cogniciones humanas (Lanier, 2018) para el beneficio de los grandes poderes económicos y políticos mundiales, o convertir la red en el escenario de nuevas formas de desarro1lo, sostenible y compartido, que surjan de la libre creatividad y sinergia humanas. Una sinergia que se da con mayor afianzamiento cuando se ejecuta con inteligencia emocional, que según Barrientos (2019) se define como "la capacidad de controlar y gestionar positivamente las emociones propias y ajenas, en un escenario cualquiera, donde se producen experiencias y cambios como parte del proceso de aprendizaje personal".

\section{Sectores de un dilema: periodismo, redes sociales, innovación empresarial}

Como explica Nogales (2020) muy recientemente, refiriéndose al mundo del periodismo, existe un dilema entre el denominado periodismo low -el periodismo que ante la llegada de la digitalización 2.0 ha generado una versión deformada y reducida de su propia actividad-, y el periodismo slow -el periodismo que, en esa misma tesitura, y una vez asentada la sociedad red, se ha regenerado con nuevas calidades, un enfoque más profundo y ético de su actividad, acorde a la función facilitadora que les atribuía Herrero (2011)-. Como indica la autora, en el periodismo low existe un "riesgo para el discurso de fortalecimiento de los derechos humanos, mientras que el periodismo slow puede favorecer su desarrollo y expansión entre la opinión pública" (Nogales, 2020:75). El dilema en el ámbito informativo es claro. Ambas líneas responden a una profunda crisis del periodismo que culminó procesos anteriores de degeneración profesional (Aladro, 2013), y de la que surgieron opciones innovadoras tras la catarsis, hacia la desindustrialización, la profundización y el compromiso ético en el periodismo. Ya desde 2011 Aladro sostenía: "El periodista actual debe ser un enlace, un selector, un editor y un evaluador, pues ya no puede erigirse en fuente ni en portavoz de la fuente" todo ello en el marco del perceptible cambio de los escenarios establecidos del que hablaban Casero, Ortells y Doménech (2014:2). En el ámbito del mundo informativo, las tendencias son claras: o el periodismo se refunda como profesión al margen de la vorágine de las fake news y la cultura de la simulación mediática, de los discursos del odio o de la manipulación política, o perece como una forma más de infoxicación digital. Resulta globalmente interesante en este 
contexto, la perspectiva aportada por Cabrera, Codina y Salaverría (2019), así como por Manfredi, Ufarte y Herranz (2019).

Voces muy similares, como las de Beling y Girardi (2018:220), que llamaban a una revisión del modus operandi periodístico en el mundo de la reflexión académica, nos indican que la web 2.0 en su desarrollo de las redes sociales y sus medios, encontramos el mismo dilema: o un crecimiento desbordante hacia su comercialización, manipulación y utilización con fines lucrativos muy minoritarios, o un avance hacia sus formas colaborativas en pro de la compartición del conocimiento, la demanda de mejores condiciones de vida y la expansión de la conciencia ecologista y medioambiental de cuidado del planeta. Así, Zuckerman (2020), es un autor profundamente versado en posibles desarrollos de las redes en la dirección adecuada, hacia el beneficio colectivo y comunitario. Sus estudios sobre el uso social de las redes comerciales como Facebook o Daily Motion, y sobre la función de los medios digitales como generadores de movimientos sociales, le han llevado a formular la necesidad de generar en las redes una infraestructura de uso social: liberar las redes sociales de sus usos puramente comerciales y lucrativos y capacitar en ellas infraestructuras digitales públicas.

Difalcis, Osiadacz y Abusamra (2018:91) nos recuerdan que la interactividad, si bien en menor grado, ya es una característica de la palabra escrita, debido al proceso plus-decodificativo señalado, entre otros por Cardoso, Bobadilla y Pérez (2018, p. 108). Del mismo modo que ocurrió con el surgimiento de los medios impresos, cuya expansión en la historia de nuestra civilización se produjo en origen de un modo desordenado, pero que fue generando una infraestructura que cubriendo el tejido social terminó por producir el libre acceso a la información, como un derecho universal, en el surgimiento del medio digital puede y debe afrontarse la idea de la necesidad de generar, como indica Zuckerman, un medioambiente digital equilibrado y benéfico, que no esté completamente instrumentalizado por el poder económico o político, algo complicado, dada la maximización de rentabilidad que, según Marta-Lazo y Gabelas (2013) traen aparejada las tecnologías móviles. La liberación de las presiones de esos aparatos de dominación supone generar, favorecer y proteger las formas en que la web 2.0 permite nuevas sinergias colaborativas y nuevas libertades de acción humana. Pero la batalla está muy lejos de ser ganada en un entorno completamente salvaje en cuanto a regulaciones eficaces, en el que se ha impuesto una auténtica batalla por el control de los datos y por el aprovechamiento condicionado de los mismos con fines exclusivamente de beneficio inmediato.

Como indica, desde Chile, Ortiz (2019), en sus leyes básicas sobre el fin que debe motivar la comunicación en el Antropoceno, "hay comunicación sí y sólo sí en un mismo tiempo y en un mismo espacio surgen entre dos o más seres humanos actos de hacer en común solidarios y altruistas en que los participantes se relacionan y lo hacen en interacciones y funciones equitativas" (2019:291). En la misma base de la comunicación interpersonal existe un entronque ético que no es posible soslayar en la era digital, e incluso, está más presente que nunca. Desde el punto de vista de la base esencial, de la raíz de la comunicación social, existe el fin altruista como motor, no solamente como embellecimiento adicional.

Las redes se nos presentan pues, como enclave en el que el miedo y/o amenaza entre el progreso humano y el beneficio individual inmediato se hace más afilada. Es en el potencial liberador de las redes donde los autores perciben con mayor claridad la necesidad de un viraje hacia una mayor función de mediación social, hasta el 
momento claramente minoritario. Los usos de las redes sociales son principalmente comerciales y publicitarios, aunque no puedan desprenderse totalmente de su valor como potenciales agentes de mediación social.

Así, los medios sociales no tienen otro sentido que el de ser mediadores sociales: como afirma Alburquerque (2015), el entorno actual tiene que favorecer el incremento de la capacidad de "pensar otras perspectivas, diferentes a las propias, y perspectivas del mundo, cosa que resulta esencial para la mayoría del mundo y las poblaciones con las cuales trabajan los mediadores sociales. Y recoge cuáles son las finalidades de las comunidades de conocimiento según Paulo Freire: construir una comprensión crítica anclada en tres ejes: el desarrollo de la consciencia colectiva, el de la consciencia social, y el de una consciencia política del sentido de los cambios.

Es especialmente flagrante el defecto con el que están funcionando las redes sociales frente a estas finalidades. En lugar de favorecer la comprensión crítica, están infoxicando con narcisismo, consumismo y complacencia social la consciencia individual, con no poca ayuda en lo tocante al consumismo, según el trabajo de Saavedra, Papí y Perlado-Lamo (2020). En lugar de desarrollar una consciencia colectiva, nos encontramos con la fragmentación absoluta de las consciencias, en redes que, por el contrario, están especialmente capacitadas para generarla, pues, como indican Cancelo y Gadea (2013:25) "a diferencia de las redes sociales físicas o tradicionales, las redes sociales en internet presentan un acceso directo entre usuarios. Destacando especialmente las acciones de comunicación directa, cooperación y creación de comunidad". Contribuyendo, según Casero (2015:2) a redefinir el activismo político y, según Camarero, Varona y Fedorov (2017) a potenciar el empoderamiento personal.

Podríamos pensar que todas estas carencias en la capacidad de crear consciencia social, crítica o política, favorecen el dinamismo de las estructuras económicas y el crecimiento vertiginoso de la iniciativa empresarial. Sin embargo, nos dicen los autores, no es así. El uso de la web 2.0 no está favoreciendo el desarrollo de un tejido empresarial innovador, equilibrado y sostenible. Ello se debe a la ventaja que los impulsos globalizadores oligopólicos están dando a la centralización de los beneficios y la restricción de las oportunidades de innovación empresarial.

Así, en los estudios sobre el potencial innovador y creativo de las iniciativas empresariales (Acosta, 2019) en medios digitales, los resultados marcan la misma brecha de la que hablamos, entre una tendencia a suprimir la innovación con finalidades sociales, que incorporen las demandas y expectativas de todos, y que realicen una gestión responsable conectada con los intereses colectivos, y la tendencia a integrarlos en el emprendimiento actual. De nuevo, el dilema entre beneficio parcial y en burbuja y desarrollo común e interconectado marca el sector de la actividad económica actual. Como indican Benfica y Barbosa (2015), la gestión, tanto social como empresarial, en los medios digitales, debe elegir entre priorizar el potencial humano, la creatividad, el respeto a las limitaciones humanas, o el incremento de la productividad.

\section{Nuevas tendencias de trabajo: las humanidades digitales, las ecologías sociales, los estudios medioambientales en la web 2.0.}

Parece claro que el mundo se enfrenta a una batalla por la elección de un modelo de desarrollo en conjunción con el desarrollo digital. Y digo se enfrenta, porque la elec- 
ción nos coloca ante situaciones y fenómenos antes nunca experimentados: el mundo de la manipulación, de las simulaciones, de los simulacros, amenaza con suplantar las verdaderas finalidades de la vida humana. Si ya Baudrillard (2017), en su clásico análisis, conectaba directamente el sistema socio-económico y la cultura del simulacro, hoy es más crucial que nunca mostrar que en la contaminación de objetivos y fines que se genera con la absoluta mercantilización de las comunicaciones humanas, la señal inconfundible es la simulación: la sustitución de la verdad por el fake, por la suplantación y por el condicionamiento reflejo que produce masivamente.

Vivimos en el momento en el que los dos caminos de futuro humano se bifurcan: uno de ellos, hacia la consecución de la verdadera finalidad humana, el impulso altruista, el cuidado y la protección de la vida en el planeta, la consecución de una sociedad más creadora e innovadora. El segundo camino, el que hemos tomado hasta el momento, nos conduce a una sociedad egoísta, narcisista y alienada a la vez, en la que la solidaridad es parcial, el condicionamiento reflejo se impone para manipular a las masas interconectadas y la innovación desaparece aplastada por los intereses de inmediato beneficio.

Es fácil comprobar que hemos tomado el segundo itinerario por las voces de alarma que entre los autores denuncian la pérdida en las posibilidades de avance en la cultura, en la educación y en el desarrollo equitativo. Una sociedad que ha optado por la no autenticidad en el desarrollo de la imagen de la mujer (Espizua y Padilla, 2017). En la que para los jóvenes "el entretenimiento está relacionado con el placer o la excitación provocados por una serie específica o por un tipo específico de series (por ejemplo, las «soap operas») ..../... se puede vincular al escapismo de la realidad“ (Fedele y García, 2010:11), en la que el narcisismo abunda en las redes adolescentes (Finol, 2014) y donde la experiencia es cada vez más simulada, más maquillada por una red social que en lugar de unir, separa.

Como indican los investigadores, la educación, la cultura y la sensibilidad provienen directamente de la experiencia. "La cultura es fundamentalmente experiencia. Y del arte interesan sobremanera aquellos fenómenos que generan lo que estos autores denominan «cadenas generativas» (Aladro et ál., 2018:11). Esas cadenas generativas se producen efectivamente en las experiencias de los jóvenes, continuando en ellos el impulso hacia la conservación y generación de la cultura, es decir, la verdadera educación. Y el medio en el que puede producirse es su medio nativo, el digital, que debe conducirnos hacia su conexión con la experiencia real en el entorno.

\section{Conclusiones}

¿Cómo concluir, entonces, nuestra disquisición, de modo que trascendamos esa bifurcación crucial de caminos, y superemos su disyuntiva? Son muchas las voces que nos indican que en la fusión de fines de diverso nivel, que es posible por el nuevo entorno digital, podemos recuperar la autenticidad de la comunicación humana y retornar hacia el modelo original de esfuerzo colectivo y creador que originó dicho entorno.

Así, Cristiano Lenzi, en su estudio sobre sostenibilidad, salud y políticas sociales (Lenzi, 2019), señala que es necesario establecer una relación directa entre estos diferentes contextos, que están interconectados mucho más de lo que pensamos, en el planeta globalizado. Las necesidades humanas, de las básicas a las de conocimiento 
o de integración natural y social, tienen que estar unidas a nuestras actividades cotidianas para aportarles un sentido. En el siglo XXI, afirma el autor, sólo la completa conexión con las dimensiones de la experiencia sensible relacionadas con el mundo natural, con el mundo social inmediato pueden aportar un sentido a la comunicación digital. Y para ello, es necesario retomar en su desarrollo como sistema comunicativo el servicio a la comunidad, y no solamente la humana, sino también la comunidad eco-social.

Blanco (2019), en su estudio sobre los retos actuales a que se enfrenta la sociedad, plantea que el único crecimiento económico posible, más ligado, si cabe que nunca, al sostenimiento de la naturaleza, pasa por dar solución a la vez a las necesidades de la población en armonía con el medio ambiente. Un escenario de post-crecimiento acelerado también para el mundo digital, en el que se comience a trabajar con la idea de potenciar una comunicación limpia, no intoxicada por su abundancia sino mesurada y difundida adecuada y equitativamente, en la que la finalidad fundamental sea la vinculación con la eliminación de las desigualdades y el conocimiento de los efectos nocivos de las iniciativas humanas, en armonía con las posibilidades innovadoras que plantean las mentes creativas, y cuya finalidad fundamental sea legar a las nuevas generaciones entornos humanitarios digitales, del mismo modo que les legamos un entorno natural protegido y fértil.

Estas conclusiones son cada vez más comunes y generalizadas. Desde los sectores del estudio de la riqueza económica potencial, de la capacidad social, de la función sinérgica e integradora de la red, que estaban en su primera fundación, en el impulso que llevó a los primeros profesionales a unir esfuerzos e iniciativas para formar la web, este debe ser el fin fundamental de la misma. Abandonar la simulación de los beneficios colectivos para abrazarlos directamente en la comunicación. Que las redes sociales, el periodismo, las plataformas emprendedoras, los sistemas culturales y educativos en este entorno, nos guíen hacia un horizonte de comunicación mediática creadora y generadora de una nueva humanidad, de un nuevo impulso social y ecológico, en la red.

\section{Bibliografía}

Acosta, Rosa (2019). Emprendimiento e Innovación: el reto de la investigación. Revista Venezolana de Gerencia, 24(85), 7-10.

Aladro-Vico, Eva (2011). La Teoría de la Información ante las nuevas tecnologías de la comunicación. CIC. Cuadernos de Información y Comunicación, 16, 83-93.[fecha de Consulta 1 de Marzo de 2020]. ISSN: 1135-7991. Disponible en: https://www.redalyc.org/ articulo.oa?id=935/93521629005

Aladro-Vico, Eva. (2013). Las teorías profesionales y las 5 crisis del periodismo. CIC. Cuadernos de Información Y Comunicación, 18, 69-81.

Aladro-Vico, Eva y Semova-Dimitrina-Bailey, Olga (2018). Artivismo: un nuevo lenguaje educativo para la acción social transformadora. Comunicar, 57, v. XXVI, 9-17.

Alburquerque, Cristina (2015). Ética do mediador social: questões críticas sobre a objectividade e a neutralidade. Mediaciones Sociales, 14, 143-160.

Barrientos-Báez, Almudena; Caldevilla Domínguez, David y García García, Enrique (2017). APP para la tercera edad: utilidad, clases y valor social. Revista de Ciencias de la Comunicación e Información, 21(2), 157-167. DOI: http://doi.org/10.35742/revistacccomunicacioneinformacion.2016.21.157-167 
Barrientos-Báez, Almudena; Caldevilla-Domínguez, David y Rodríguez-Terceño, José (2020). Adaptación educativa a partir del análisis de las guías docentes del grado universitario en turismo. Revista Fronteiras: Journal of Social, Technological and Environment Science.

Baudrillard, Jean (2017). Cultura y simulacro. Barcelona: Kairós.

Beling Loose, Eloisa y Girardi, Ilza Maria Tourinho (2018). Antes do desastre: notas a respeito do Jornalismo, da comunicação de riscos, da prevenção e do envolvimento cidadão. Mediaciones Sociales, 17, 209-222.

Benfica, Alva y Barbosa, Iris (2015). Contribuçôes da neurociencia para a gestâo de pessoas. Opción, 31, 113-133.

Blanco-Obando, Edgar (2019). Medio ambiente y desarrollo: resultados ambientales y sociales de la operación de las mayores actividades productivas en la región Atlántico/Caribe de Costa Rica, 1990-2015. Revista de Ciencias Sociales, 64.

Cabrera Méndez, Marga; Codina, Lluís y Salaverría Aliaga, Ramón (2019). Qué son y qué no son los nuevos medios. 70 visiones de expertos hispanos. Revista Latina de Comunicación Social, 74, 1506-1520. http:/www.revistalatinacs.org/074paper/1396/79es.html DOI: $10.4185 /$ RLCS-2019-1396-79

Camarero, Emma; Varona, David y Fedorov, Alexander (2017). Alfabetización Mediática y Audiovisual para el Empoderamiento y el Cambio Social: Resultados de Proyecto Nica (1 $1^{\circ}$ Fase). Opción, 33(82), 160-189.

Cancelo-Sanmartín, Mercedes y Gadea-Aldave, Gilda (2013). Empoderamiento de las redes sociales en las crisis institucionales. Vivat Academia. Revista de Comunicación, 15(124), 21-33. DOI: http://dx.doi.org/10.15178/va.2013.124.21-33

Cardoso, Daniel; Bobadilla, Salvador y Pérez, Manuel Antonio (2018). Habits of reading in college. Case degree of administration of the Tejupilco professional academic unit. Investigaciones Sobre Lectura, 9, 73-104.

Casero-Ripollés, A. (2015). Estrategias y prácticas comunicativas del activismo político en las redes sociales en España. Historia Y Comunicación Social, 20(2), 533-548. https:// doi.org/10.5209/rev_HICS.2015.v20.n2.51399

Casero-Ripollés, Andreu; Ortells-Badenes, Sara y Doménech-Fabregat, Hugo (2014). Las competencias profesionales en periodismo: una evaluación comparativa. Historia y Comunicación Social, 18, 53-64. https://doi.org/10.5209/rev_HICS.2013.v18.44311

Castells, Manuel (2005). La Era de la Información. La Sociēad Red. Alianza Editorial.

Difalcis, M., Ferreres, A., Osiadacz, N. y Abusamra, V. (2018). Reading response latencies in Spanish: effects of lexicality and frequency. Investigaciones Sobre Lectura, 9, 50-72.

Espizua, Inmaculada y Padilla-Castillo, Graciela (2017). La imagen y el estilo de la mujer política española como elementos básicos de su comunicación. Revista de Comunicación de la SEECI, 21(42), 62-84.

Fedele, Maddalena y García-Muñoz, Nuria (2010). El consumo adolescente de la ficción seriada. Vivat Academia. Revista de Comunicación, 111, 47-64.

Finol, Enrique (2014). Nuevos escenarios de la corposfera: Fotografía, selfies y narcisismo. Letra, imagen y sonido, 6(11).

Gómez Nieto, Begoña; Tapia Frade, Alejandro y Díaz Chica, Oscar (2012). La comunicación corporativa a través de las páginas web: el caso de las ONGs españolas. Vivat Academia. Revista de Comunicación, 14(120), 1-20. DOI: http://dx.doi.org/10.15178/ va.2012.120.1-20

Herrero-Curiel, E. (2011). El periodismo en el siglo de las redes sociales. Vivat Academia. Revista de Comunicación, 117E, 1113-1128. DOI: http://dx.doi.org/10.15178/ 
va.2011.117E.1113-1128

Lanier, Jaron (2018). Diez razones para borrar tus redes sociales. Barcelona: Debate.

Lenzi, Cristiano Luis (2019). Sustentabilidade, Saúde E Políticas Sociais: Repensando O Bem-Estar Social No Século 21. Fronteiras: Journal of Social, Technological and Environmental Science, 8(3), 338-55.

Lessig, Lawrence (2009). El código 2.0. Madrid: Traficantes de sueños.

Lopezosa, Carlos; Codina, Lluís; López-García, Guillermo y Corbella-Cordomi, Juan-María (2020). Mapa de visibilidad y posicionamiento en buscadores de los principales grupos mediáticos españoles. El profesional de la información, 29(2), e290203. DOI: https://doi. org/10.3145/epi.2020.mar.03

Manfredi Sánchez, Juan Luís; Ufarte Ruiz, María José y Herranz de la Casa, José María (2019). Innovación periodística y sociedad digital: Una adaptación de los estudios de Periodismo. Revista Latina de Comunicación Social, 74, 1633-1654. http://www.revistalatinacs.org/074paper/1402/85es.html DOI: 10.4185/RLCS-2019-1402

Marta-Lazo, Carmen y Gabelas-Barroso, José Antonio (2013). Hábitos de consumo televisivo de ficción entre los universitarios que estudian Comunicación. Revista de Comunicación de la SEECI, 17(31), 14-33. DOI: http://dx.doi.org/10.15198/seeci.2013.31.14-33

Nogales, María Antonia (2020). Periodismo low, periodismo slow y derechos humanos. Diferencias y riesgos en la cobertura informativa del fenómeno migratorio en España. Inclusiones, Revista de Ciencias Sociales y Humanidades, 7(2), 75-103.

Ortiz-Veas, Manuel (2019). Valores y Comunicología en el Antropoceno y Robotecnológeno. Perspectivas de la Comunicación, 12(2) 281-292.

Saavedra-Llamas, Marta; Papí-Gálvez, Natalia y Perlado-Lamo-de-Espinosa, Marta (2020). Televisión y redes sociales: las audiencias sociales en la estrategia publicitaria. El profesional de la información, 29(2), e290206. DOI: https://doi.org/10.3145/epi.2020.mar.06

Sánchez Fuentes, Sergio y Martín Almaraz, Rosario Ángela (2016). Formación docente para atender a la diversidad. Una experiencia basada en las TIC y el diseño universal para el aprendizaje. Revista de Ciencias de la Comunicación e Información, 21(1), 35-44. DOI: http://doi.org/10.35742/revistacccomunicacioneinformacion.2016.21.35-44

Sánchez Limón, Mónica Lorena y De la Garza Cárdenas, Manuel Humberto (2018). Tecnologías de información y desempeño organizacional de las pymes del noreste de México. Revista Venezolana de Gerencia (RVG) 23(82), 298-313.

Zuckerman, Ethan (2020). The Case for Digital Public Infrastructure. Harnessing past successes in public broadcasting to build community-oriented digital tools. Disponible en https://knightcolumbia.org/content/the-case-for-digital-public-infrastructure 